Les stratégies déployées par les apprenti·e·s en commerce de détail pour faire face aux conditions de travail difficiles en Suisse

Strategies used by trainees in the retail sector to cope with difficult working conditions in Switzerland

Las estrategias implementadas por los aprendices en comercio minoristas para hacer frente a las difíciles condiciones laborales en Suiza

Isabelle Caprani, Kerstin Duemmler et Alexandra Felder

Volume 22, numéro 2, 2020

URI : https://id.erudit.org/iderudit/1074420ar

DOI : https://doi.org/10.7202/1074420ar

Aller au sommaire du numéro

Éditeur(s)

Faculté d'éducation, Université de Sherbrooke

ISSN

1911-8805 (numérique)

Découvrir la revue

Citer cet article

Caprani, I., Duemmler, K. \& Felder, A. (2020). Les stratégies déployées par les apprenti.e-s en commerce de détail pour faire face aux conditions de travail difficiles en Suisse. Nouveaux cahiers de la recherche en éducation, 22(2), 1-20. https://doi.org/10.7202/1074420ar
Résumé de l'article

Cette contribution se base sur une recherche auprès d'apprenti·ess en commerce de détail en Suisse et étudie leur manière de répondre aux exigences et contraintes du métier. Il s'agit de relever à quelles stratégies elles et ils ont recours pour faire face aux conditions de travail pénibles. En se référant au concept du coping, trois principales stratégies ressortent de l'analyse : résistance, passivité et autonomie. Déterminer les stratégies pour répondre aux situations de travail et de formations difficiles permet de mieux comprendre la manière dont les apprenti·e-s développent et renforcent leur rapport au métier. 


\title{
Les stratégies déployées par les apprenti-e.s \\ en commerce de détail pour faire face aux conditions de travail difficiles en Suisse
}

\author{
Isabelle Caprani \\ Kerstin Duemmler \\ Alexandra Felder
}

Institut fédéral des hautes études en formation professionnelle (IFFP)

\section{Résumé}

Cette contribution se base sur une recherche auprès d'apprenti·e·s en commerce de détail en Suisse et étudie leur manière de répondre aux exigences et contraintes du métier. Il s'agit de relever à quelles stratégies elles et ils ont recours pour faire face aux conditions de travail pénibles. En se référant au concept du coping, trois principales stratégies ressortent de l'analyse: résistance, passivité et autonomie. Déterminer les stratégies pour répondre aux situations de travail et de formations difficiles permet de mieux comprendre la manière dont les apprenti-e.s développent et renforcent leur rapport au métier.

Mots-clés: conditions de travail, stratégies de coping, rapport au métier, formation professionnelle, apprenti·e.s, Suisse 


\section{Strategies used by trainees in the retail sector to cope with difficult working conditions in Switzerland}

\section{Abstract}

This article based on a study conducted with trainees in the Swiss retail sector examines how they cope with the demands and constraints of the industry. The strategies employed to deal with arduous working conditions are outlined. With respect to the concept of coping, three main strategies were gleaned from the data: resistance, passivity and autonomy. Identifying the strategies used by trainees to cope with difficult working and training conditions provides insight into how trainees develop and strengthen their relationship with the occupation.

Keywords: working conditions, coping strategies, relationship with the profession, professional development, trainees, Switzerland

\section{Las estrategias implementadas por los aprendices en comercio minoristas para hacer frente a las difíciles condiciones laborales en Suiza}

\section{Resumen}

Esta contribución se basa en una investigación ante aprendices de comercio minorista en Suiza y estudia cómo satisfacen las demandas y limitaciones de la profesión. Se trata de identificar qué estrategias utilizan para hacer frente a las duras condiciones laborales. Refiriéndonos al concepto de afrontamiento, surgen tres estrategias principales del análisis: resistencia, pasividad y autonomía. Determinar las estrategias para dar respuesta a situaciones laborales y formativas difíciles, nos permite comprender mejor la forma en que los aprendices se desarrollan y fortalecen su relación con la profesión.

Palabras clave: condiciones de trabajo, estrategias de afrontamiento, relación con el trabajo, formación profesional, aprendices, Suiza 


\section{Introduction}

Les évolutions constantes du marché du travail, qui ont conduit à une dérégulation et une flexibilisation de l'emploi telles que le contrat de travail à durée déterminée ou le travail à temps partiel, tendent à fragiliser l'accès à l'emploi particulièrement pour les jeunes en début de formation'1 (Askenazy, Behaghel, Laouenan et Meurs, 2019, Salvisberg, 2012; Voirol-Rubido et Hanhart, 2015). Ces dernières et derniers sont également confrontés à des exigences accrues et des changements constants en matière de qualifications et compétences recherchées (Mercure et Vultur, 2010; Tomlinson, 2013). Ces changements influencent le rapport au métier, poussant les individus à se battre pour se faire une place sur le marché du travail devenu plus concurrentiel; l'emploi sûr devient dès lors une denrée rare (Bauman, 1998; Billett, 2006, Tomlinson, 2013). Face à ces difficultés rencontrées au moment de l'entrée en formation, mais également tout au long de l'apprentissage ${ }^{2}$, les apprenti·e.s vont devoir trouver des façons de gérer des conditions et situations difficiles pour pouvoir s'insérer dans le monde du travail.

Cette contribution qui se base sur une étude auprès d'apprenti·e·s gestionnaires en commerce de détail en Suisse propose d'étudier la manière dont ces jeunes en formation font face aux exigences et contraintes dans leur métier et le monde du travail en général. Malgré des conditions de formation et de travail plutôt difficiles, telles que les longues journées de travail, les horaires irréguliers, le travail les week-ends/soirées, la répétitivité des tâches (Barbier, 2012a, 2012b; Price, 2011; Williams et Connell, 2010), et précaires, dues aux bas salaires ou au travail à temps partiel (Angeloff, 2000), les apprenti·e.s en commerce de détail ne développent pas nécessairement un rapport négatif à l'égard de leur métier (Caprani, Duemmler et Felder, 2019). Ces jeunes en formation chercheront plutôt des arrangements dans le but d'améliorer leur quotidien. Nous montrerons qu'en adoptant des réactions variées elles et ils s'investissent et s'intègrent de diverses façons dans leur métier. Il s'agira donc de relever à quelles stratégies les apprenti·e·s ont recours pour déterminer les modes d'investissement professionnel (Osty, 2003) et la manière dont elles et ils arrivent à les utiliser pour mieux vivre leurs conditions de travail difficiles. Pour ce faire, nous baserons nos analyses sur les stratégies comportementales et cognitives qui peuvent être mises en lien avec le concept de coping (Lazarus et Folkman, 1984; Voss, 2002). Le coping, qui se présente comme un modérateur de la relation événement stressant/ détresse émotionnelle, répond à une situation difficile, en l'occurrence la réalité du travail à laquelle il est crucial de faire face pour réussir sa formation (Lazarus et Folkman, 1984).

$1 \quad$ L'âge d'entrée en formation en Suisse est de 15 ans révolus.

2 À noter qu'en Suisse le cursus en formation professionnelle est appelé «apprentissage» et les jeunes en formation sont désignés par l'appellation "apprenti·e·s". 
Afin de comprendre l'influence des conditions de travail dans la formation des apprenti.e.s, le contexte de la formation en commerce de détail et les difficultés du métier auxquelles les jeunes en formation sont confrontés dans leur quotidien seront exposés dans un premier temps. Nous définirons dans un second temps le cadre théorique qui s'appuie sur les stratégies du coping ainsi que les méthodes d'enquête retenues pour mener cette étude qualitative. Nous procéderons ensuite à l'analyse des principales stratégies mises en place par les apprenti.e.s pour répondre aux conditions de travail. La discussion mettra en lumière le fait que le rapport qu'elles et ils nouent avec leur métier est étroitement lié à leur manière de réagir face aux difficultés rencontrées sur le lieu de travail.

\section{Contexte de la formation en alternance en commerce de détail}

Gestionnaire en commerce de détail est la troisième plus importante formation professionnelle en Suisse. En 2019, sur près de 66000 nouveaux contrats d'apprentissage, on dénombre 4395 apprenti.e.s inscrite.es en commerce de détail dont $60 \%$ de femmes (SEFRI, 2020). Le cursus de formation implique la vente, le conseil à la clientèle et la gestion de la marchandise. D'une durée de trois ans, la formation se déroule en école professionnelle durant 1,5 jour par semaine, et les apprenti-e.s travaillent en entreprise pendant 3,5 jours dans différents domaines de la vente tels que les grandes surfaces alimentaires, les épiceries, les magasins de sport, les bijouteries, les parfumeries, etc. À cela s'ajoutent dix jours par année de cours interentreprises qui permettent la mise en pratique des savoirs professionnels acquis.

Souvent sous-estimé et considéré comme un métier faiblement qualifié (Bozkurt et Grugulis, 2011; Price, 2011; Roberts, 2012; William et Connell, 2010), gestionnaire en commerce de détail nécessite pourtant diverses compétences d'un travail de service en particulier dans l'interaction quotidienne avec la clientèle faisant appel à des aptitudes à la communication, à la gestion émotionnelle ainsi qu'à la connaissance et expertise des produits (Gatta, 2011). Le contexte économique exigeant se caractérise par une concurrence en constante évolution voire en augmentation. L'installation de magasins étrangers à bas prix, le shopping touristique dans les pays étrangers limitrophes proposant souvent des produits moins chers ou les petits magasins des centres-villes concurrencés par les grandes surfaces en périphérie en constituent des exemples. L'apparition de nouvelles formes de distribution telles que le commerce en ligne (Benoun, 2015) engendre aussi un accroissement des compétences demandées (Cianferoni, 2019). L'intensité du travail mêlée aux bas salaires, au temps partiel ainsi qu'au manque de prestige du métier entraînent d'importants problèmes aussi bien pour les employée.es que les entreprises notamment sur le plan du recrutement (Cianferoni, 2019; Duemmler, Felder et Caprani, 2016). Les 
horaires de vente sont aussi de plus en plus libéralisés. Travailler dans le commerce de détail implique des horaires de travail irréguliers et de longues journées fragmentées y compris les soirs et les week-ends (UNIA, 2012) avec toutes les conséquences négatives que cela suppose dans les domaines de la vie privée (Quiénnec, Gadbois et Preteur, 1995). Ces différents facteurs rendent les conditions de travail toujours plus exigeantes tout en précarisant la situation des employée.es. Dans ce contexte, difficile de prévoir l'évolution du métier et des besoins en main-d'œuvre qui en découleront (Benoun, 2015).

Les apprenti-e.s en commerce de détail de la présente recherche ont également évoqué ce contexte pénible du travail en entreprise (Caprani, Duemmler et Felder, 2019). Parfois ces jeunes en formation ont le sentiment d'être exploités, en raison notamment d'une forte concurrence et d'une pression propre au secteur de la vente (Bozkurt et Grugulis, 2011; Price, 2011; Roberts, 2012; William et Connell, 2010). Or, ces contraintes peuvent les pousser à adopter différentes réactions et comportements pour faire face aux conditions de travail difficiles qui peuvent se traduire par une nécessité de mettre en œuvre des stratégies de coping; c'est ce que nous étudierons dans les points suivants.

\section{Cadre théorique: le rôle des stratégies de coping dans le développement du rapport au métier}

Pour mener cette étude sur la manière de faire face aux conditions de travail difficiles, nous nous sommes référées à une conceptualisation du coping. Ce concept a été à l'origine élaboré par Lazarus et Launier en 1978 et ensuite repris et développé par nombre d'auteurs (Billings et Moos, 1981; Coyne, Aldwin et Lazarus, 1981; Endler, 1988; Endler et Parker, 1990; Mariage, 2001; Pearlin et Schooler, 1978, Ray, Lindop et Gibson, 1982). Lazarus et Folkman (1984) définissent le coping comme «l'ensemble des efforts cognitifs et comportementaux constamment changeants (mise en œuvre) pour gérer des demandes externes et/ou internes spécifiques qui sont évaluées comme menaçant ou excédant les ressources de la personne" (p. 141). Les auteurs partent ainsi de l'idée que durant sa vie l'individu est confronté à des événements qu'il peut percevoir comme menaçants, induisant par là même des perturbations émotionnelles. La menace ne pouvant être tolérée très longtemps, l'individu cherchera des arrangements par le développement de stratégies de coping qui auront comme objectif de répondre à une situation de contraintes professionnelles ou de stress.

À l'origine, le coping est affilié aux réflexions sur les concepts de mécanisme de défense et sur celui d'adaptation. Le premier, comme le relève Bruchon-Schweizter (2001), a été initialement pensé comme "rigide, inconscient, indifférencié, irrésistible, lié à des conflits intra-psychiques et des événements de vie anciens" (p. 69). L'approche traditionnelle de la stratégie de la défense envisage le coping comme un trait de personnalité permettant de 
rétablir l'équilibre émotionnel avec comme dessein la réduction de la tension plutôt que la résolution du problème (Paulhan, 1992). Cette conception fut fortement critiquée, dans le sens que l'individu ne se comporte pas toujours de manière identique face au stress. Les stratégies qui en découlent sont au contraire flexibles, conscientes et différenciées, et permettent à l'individu de maîtriser, réduire ou supporter les perturbations induites par une situation problématique (Bruchon-Schweitzer, 2001). Bruchon-Schweitzer, qui a tenté une synthèse de la conséquente littérature sur le sujet, relève que ces stratégies de coping se distinguent des mécanismes de défense, car ce sont des tentatives conscientes et donc volontaires pour affronter des problèmes actuels ou récents. Ce type de stratégies est ainsi orienté vers la réalité dans le but d'atténuer les tensions internes et externes (Chabrol, 2005). Le coping se réfère aussi au mécanisme prenant sa source dans les théories relatives à l'adaptation ${ }^{3}$. Ces dernières ont la particularité d'intégrer les modes de réaction des organismes vivants qui interagissent avec les conditions changeantes de l'environnement. Le coping est en revanche plus spécifique puisqu'il intègre des réactions à des variations de l'environnement définies comme menaçantes. Il implique ainsi également des comportements conscients, changeants, précis et parfois nouveaux pour l'individu (Bruchon-Schweitzer, 2001).

Le modèle de coping qui semble se distinguer est celui basé sur l'approche transactionnelle qui prend en compte l'interaction entre la personne et son environnement (Bruchon-Schweitzer, 2001). Pour faire face à une situation stressante ou menaçante dans le but de modifier sa relation avec l'environnement, l'individu va évaluer les ressources et réponses dont il dispose. Dans ce type d'approche, le coping exerce deux fonctions principales. La première est une manière de gérer, atténuer, voire agir sur le problème (Nicchi et Le Scanff, 2005). Cette stratégie permet de modifier directement les caractéristiques de la relation personne/environnement par la mise en place d'efforts comportementaux actifs de confrontation au problème dans le but de le résoudre (Paulhan, 1992). La deuxième stratégie se focalise sur l'émotion, avec l'objectif de réguler la réaction émotionnelle (Nicchi et Le Scanff, 2005). Une possibilité implique la modification de l'attention en se détournant de la source de stress par une stratégie d'évitement. L'une des plus courantes, cette stratégie peut être interprétée comme un moyen de se dégager, physiquement et psychologiquement, d'une situation sous stress (Endler et Parker, 1990). Par l'intermédiaire d'activités de substitutions comportementales et cognitives telles que les activités sportives, jeux, relaxation, loisirs, elle permet de se débarrasser de la tension émotionnelle, ce qui va aider l'individu à se sentir mieux (Paulhan, 1992). Suls et Fletcher (1985), qui se distancient de la classification de Lazarus et Folkman, distinguent le coping d'évitement qui opposeraient des stratégies passives - telles que évitement, fuite,

3 À noter que la littérature scientifique française parle également de "stratégies d'ajustement" (Dantchev, 1989; Dantzer, 1989). 
déni, acceptation - au coping de vigilance qui implique des stratégies actives - comme la recherche d'informations, le soutien social, les plans de résolution de problème. Le coping est ici défini comme une stratégie multidimensionnelle de contrôle dans le but de changer la situation menaçante (Dantchev, 1989).

Différents auteurs ont ajouté à ces stratégies la prise en compte de la recherche du sens - meaning-focused coping - comme un autre moyen d'adaptation, dans l'idée que donner du sens à une situation menaçante permet de mieux la vivre (Folkman et Lazarus, 1980; Folkman et Moskowitz, 2004; Park et Folkman, 1997). En prenant appui sur ses valeurs et croyances, l'individu modifierait ainsi le sens d'une transaction stressante (Folkman et Moskowitz, 2004) pour l'affronter et ne pas se laisser submerger.

Dans notre analyse portant sur les apprenti-e.s en commerce de détail, nous avons pris en compte l'ensemble de ce cadre théorique sur le coping. Ainsi, les stratégies pour faire face aux conditions de travail difficiles qui émergent de l'analyse font largement écho à ces différentes réflexions. Nous allons voir qu'une première stratégie, proche du mécanisme de défense, confronte et tente de résoudre le problème. Une deuxième, proche du mécanisme d'adaptation, vise la relativisation de la situation au niveau cognitif pour mieux gérer les émotions. Une troisième, relevant également d'un mécanisme d'adaptation, permet de donner du sens à la situation et de s'y adapter.

Cette analyse du développement de stratégies par les apprenti.e.s pour répondre à des situations de travail et de formation difficiles est apparue comme une démarche adaptée dans le but de mieux comprendre la manière dont elles et ils développent leur rapport au métier et au travail quotidien. Le rapport au métier comporte à la fois l'acquisition du savoir-faire théorique et pratique, mais également du savoir-être associé à l'appropriation de règles, de normes, de codes et de valeurs du métier. Colley, James, Diment et Tedder (2003), tout en s'appuyant sur le concept clé de Bourdieu (1980), parlent même d'habitus professionnel pour étudier l'acquisition de ces savoirs et le rapport que les apprenti-e.s doivent développer pour être en phase avec leur champ professionnel. Mercure et Vultur (2010) se sont intéressés plus concrètement au rapport au travail qui se manifeste par "une satisfaction ou insatisfaction des travailleurs quant au contenu du travail, aux conditions de travail et aux relations sociales au travail» (p. 23). Dans notre cadre d'analyse, cette définition est tout à fait pertinente, puisqu'elle implique une réponse des apprenti-e.s à l'égard notamment des conditions de travail. Nous allons ainsi voir que les stratégies adoptées par les apprenti·e·s influencent leur rapport au métier. 


\section{Méthodologie}

Cet article s'appuie sur un large projet de recherche qualitative qui étudie le développement et la négociation des identités professionnelles des apprenti·e·s durant leur formation. Notre analyse qui se base sur des données recueillies entre 2015 et 2016 a été menée au sein de quatre classes de trois écoles professionnelles suisses en commerce de détail, à savoir trois classes de première année et une classe de troisième année.

Afin de mieux comprendre le rapport que développent les jeunes en formation avec leur métier et leur travail, une démarche qualitative basée sur des entretiens et discussions de groupe a été privilégiée. Nous avons, dans un premier temps, mené des observations en classe et durant les pauses, comptabilisant 12 jours par classe, dans le but de mieux connaître les contextes de formation des apprenti·e.s. Cette démarche méthodologique a permis aussi bien de créer un climat de confiance avec les apprenti·e·s que d'affiner notre canevas d'entretien en relevant les thématiques à approfondir. Nous avons mené ensuite 25 entretiens semi-directifs avec des apprenti·e·s âgés de 16 à 22 ans dont 11 hommes et 14 femmes. Nous les avons interrogés sur leurs expériences en entreprise, leur rapport à la tâche, les conditions de travail, les relations sociales au sein de l'entreprise et leurs aspirations futures. Cela a permis d'obtenir une critique indirecte des situations vécues au sein de leur métier. En s'exprimant sur leur situation de formation en entreprise et les stratégies qu'elles et ils ont pu adopter, les apprenti·e·s ont ainsi pu présenter leurs expériences en entreprise ainsi que les difficultés rencontrées. Cette démarche a permis aussi de les faire réfléchir sur les savoir-faire et savoir-être développés dans le cadre de leur expérience en entreprise.

À la suite de cette enquête par observation et entretiens, dix discussions de groupes focalisés avec des apprenti-e.s ont été effectuées, dans un premier temps au sein des quatre classes participant à l'étude, ce qui a permis de discuter de leurs conditions de formation. Puis, de nouvelles discussions de groupes focalisés ont été menées en fin d'études auprès de l'ensemble des classes de dernière année des deux écoles, à savoir six classes de troisième année dans le but de discuter des premiers résultats d'analyse. Ces secondes discussions ont également permis d'approfondir les entretiens menés en amont et de relever l'évolution des apprenti·e·s, eu égard à leur formation et leur rapport au métier.

Le parcours scolaire de ces apprenti-e.s pour la plupart non linéaire, soit 21 des 25 jeunes en formation, se caractérise par un passage dans une structure de transition après l'école obligatoire pour trouver une place d'apprentissage ou dans une autre formation. À noter que neuf apprenti·e·s ont connu un arrêt d'apprentissage. La majorité était scolarisée dans une filière à bas niveau d'exigence durant l'école obligatoire, ce qui restreint les possibilités de choix de formation (Meyer et Bertschy, 2011). D'ailleurs, la formation en commerce de détail n'était pas, pour la majorité d'entre elles et eux, leur 
premier choix professionnel. Quant à leurs parents, ils sont essentiellement issus de la classe ouvrière ou de la classe moyenne inférieure. À l'image de la moyenne nationale, un quart des apprenti.e.s interrogée.e.s sont issu.e.s de l'immigration des première et deuxième générations (ex-Yougoslavie, Turquie, Portugal, Italie, France et Allemagne).

Le matériel empirique a été entièrement retranscrit, à savoir les notes d'observation, les entretiens et discussions de groupes focalisés enregistrés. Une analyse par codage - inspirée de la méthodologie développée par la théorisation ancrée (Charmaz, 2001; Glaser et Strauss, 1967) - a été retenue afin d'être proche des préoccupations des apprenti.e.s. Un codage ouvert, mais sensibilisé par le cadre théorique des stratégies du coping a été privilégié, ce qui a permis de relever lors de l'analyse de données différentes stratégies de ces jeunes en formation face aux conditions de travail. Les difficultés de faire face aux conditions de travail sur le lieu de formation en entreprise ont rapidement émergé de l'étude ethnographique de terrain. Elles sont d'ailleurs ressorties de manière spontanée comme thème aussi bien lors des discussions de groupes focalisés que durant les entretiens.

\section{La mise en place de stratégies du coping pour faire face aux conditions de travail}

Le commerce de détail se caractérise par des conditions de travail pénibles (Barbier, 2012a, 2012b; Price, 2011; Williams et Connell, 2010). Une première analyse de l'expérience subjective des conditions de travail a permis de faire ressortir quatre dimensions expérimentées par les apprenti-e·s de la présente étude: les exigences et l'intensité du travail, la disponibilité temporelle et l'insécurité socio-économique, le soutien et les rapports sociaux, ainsi que le rapport à la clientèle (Caprani, Duemmler et Felder, 2019). Nous nous sommes appuyés sur le concept de coping pour analyser les stratégies des apprenti.e.s pour y faire face. En cherchant des arrangements, ces dernières et derniers ont comme dessein de rendre leur situation de formation en entreprise plus agréable ou tout au moins acceptable. Trois stratégies dominantes reflétant la récurrence de leurs comportements face à des situations problématiques ont émergé des analyses.

\subsection{Stratégie de résistance: un moyen d'améliorer sa place au sein de l'entreprise}

Une première stratégie, la résistance, qui se rapproche du mécanisme de défense, va permettre aux apprenti.e.s de se protéger et mieux supporter leur contexte de formation en entreprise. Dans nos analyses, nous avons relevé une récurrence de stratégies défensives de la part de plusieurs apprenti-e.s en réponse aux conditions difficiles de travail en entreprise. En agissant sur leur environnement de travail, ces jeunes en formation adoptent des réactions de refus ou d'opposition dans la perspective de se 
protéger face à une situation problématique que nous définissons comme une stratégie de résistance.

Face à une attitude désagréable de client-e-s perçue comme une agression, des apprenti-e.s peuvent adopter une stratégie de résistance en refusant par exemple de les servir. Leila - apprentie dans un magasin spécialisé en matériel de construction -, qui déclare avoir subi des remarques à caractère sexuel d'un client, refuse de le servir et demande à ses collègues de s'en charger. Par son refus, elle adopte une stratégie de résistance dans le but de réduire les perturbations que cela pourrait engendrer pour elle et se protège ainsi d'une éventuelle nouvelle agression.

La stratégie de résistance est adoptée dans des situations précises, par exemple lorsque des apprenti.e.s se trouvent confrontée.es à un conflit de valeur que Coutrot et Mermilliod (2010) définissent comme un état de mal-être ressenti lorsque le travail demandé est contradictoire vis-à-vis des normes professionnelles, sociales ou subjectives. C'est le cas de Myriam - apprentie en boucherie et poissonnerie - qui refuse la demande de son patron, à savoir mentir à la clientèle sur la fraîcheur de la marchandise:

Je trouve ça pas honnête. Mon patron, il dit tout le temps: «Faut dire qu'il est de ce matin le poisson, sinon les gens ils croient qu'il est pas frais du tout.» [...] Donc vous pouvez m'engueuler, tout ce que vous voulez, moi je tiens pas compte de vos remarques, je continuerai à travailler comme je travaille, parce que moi je trouve ça pas normal.

Dans ce contexte, la stratégie de résistance ne pourra pas résoudre en soi le problème, puisque le désaccord avec son patron demeure. Même si nous situons notre analyse du point de vue des apprenti.e.s, un véritable enjeu de pouvoir peut s'instaurer entre la personne encadrante et son apprenti.e. En effet, la relation de formation implique aussi une relation de pouvoir qui peut se transformer en lutte (Gadrey, Jany-Catrice et PernodLemattre, 2005; Caprani, Duemmler et Felder, 2019).

Pour défendre ses intérêts et valeurs, ce qui peut aller à l'encontre des directives de l'entreprise ou parfois même des collègues ou de la clientèle, les apprenti·e.s doivent faire preuve de beaucoup de détermination. Yanis - apprenti en magasin de téléphonie - qui déplore le changement constant de ses heures de travail a exigé auprès de son supérieur de le libérer chaque jeudi soir pour suivre un cours de danse:

J'ai commencé à danser vers le mois de décembre, et puis je leur ai dit: «Écoutez, j'ai commencé à danser, c'est le jeudi soir, et je serais heureux si je pouvais faire le service du matin.» [...] Ensuite le patron a dit: "Oui, je ne peux pas vous promettre que c'est tous les jeudis." Etj'ai dit: "Non, je paie pour cetteformation et sinon je veux juste un salaire plus élevé en tant qu'apprenti» ou quelque chose comme ça. $O K$, c'était un peu prétentieux à l'époque, mais heureusement je le connais depuis trois ou quatre ans maintenant, c'est un bon collègue, et donc nous ne nous sommes pas pris trop au sérieux, mais, maintenant, je fais le service du matin tous les jeudis. 
Yanis a finalement réussi à s'imposer, notamment grâce au soutien de son formateur. Il a ainsi affronté le problème dans le but de le résoudre. Avoir le courage de défendre ses intérêts dépend également de la culture d'entreprise qui prend en compte, ou pas, les besoins de ses employéee's et apprenti.e.s. En répondant positivement à la demande de Yanis, l'entreprise a contribué à renforcer son sentiment d'appartenance puisqu'il se décrit comme étant fier de travailler pour une entreprise avec de bonnes conditions de travail.

L'analyse des entretiens d'apprenti-e.s de troisième année ainsi que de la deuxième série de groupes focalisés montre que les jeunes de dernière année de formation osent davantage s'opposer à leur hiérarchie et ont ainsi plus souvent recours à ce type de stratégie. Nous émettons l'hypothèse que par l'expérience acquise durant leurs trois années de formation, les apprenti.e.s se sont familiarisé.e.s avec les règles du métier et ont ainsi pu davantage développer la confiance en elles et eux. Karim - apprenti dans une grande enseigne commerciale - l'exprime ainsi:

\begin{abstract}
Avec une voix simple, juste le dire. Parce que maintenant si ma cheffe elle me dit un truc, et j'approuve pas. Je lui dis: "C'est ton droit, moi j'approuve pas" et il n'y a pas de soucis. Mais si je dis rien, moi je vais ruminer et le soir je vais être mal, elle m'a dit ça et j'ai rien dit. Si j'avais 16-17 ans peut-être que je serais toujours à dire non et tout, là justement j'en ai 22 et depuis trois-quatre ans je suis comme ça, doncj'ai peut-être de la chance. Moi, j'encourage tous à faire ça sinon tu te fais bouffer. Parce qu'ils se parlent entre eux, après ils disent: "Lui, il accepte tout, donc c'est bon tu vas pouvoir bouffer sur lui.»
\end{abstract}

En agissant de la sorte, Karim pense à son bien-être au sein de l'entreprise, puisqu'il anticipe sur ses futures relations de travail. Sa réaction illustre fort bien cette première stratégie de résistance dont l'objectif est de maîtriser une situation problématique en modifiant directement la relation personne/environnement. II intègre ainsi une stratégie de résistance dans l'intention d'anticiper le problème.

\title{
5.2 Stratégie de passivité: une manière de mieux vivre sa formation en entreprise
}

Une deuxième stratégie développée par les jeunes en formation se définit par une relative passivité. Elle relève d'un mécanisme d'adaptation à une situation problématique dans le but de s'y accommoder. À la différence de la stratégie de résistance, elle ne vise pas à agir sur le contexte, mais se manifeste par une relativisation de la situation sur le plan cognitif dans le but de mieux gérer son émotion et ainsi de réduire la tension. Même si ces jeunes en formation n'y adhèrent pas forcément, elles et ils sont nombreuses et nombreux à accepter les conditions de travail au sein de leur entreprise. Ces dernières et derniers s'y adaptent par une sorte de conformisme imposé avec comme but principal de mener leur formation à son terme. 
En réponse à l'impolitesse de certain·e·s client·e.s, la prise de distance, afin de ne pas se laisser submerger par ce manque de considération, apparaît comme une stratégie d'adaptation efficace. Myriam - apprentie en boucherie et poissonnerie - l'exprime ainsi:

Déjà que moi des fois comme les clients, ils parlent, faut vraiment être..., faut laisser passer en fait. Au début c'est très dur franchement, moi j'ai un bon caractère, et les gens qui disent pas bonjour, qui disent pas merci ou comme ça, moi ça m'énerve. Maintenant ça va! J'ai réussi à passer au-dessus.

Ici, Myriam, qui n'est pas en accord avec le comportement de la clientèle, développe un savoir-faire pratique de métier qu'elle pourra ainsi appliquer dans d'autres situations similaires. Dans certains cas, la stratégie de passivité devient même une technique de protection à l'égard d'une attitude interprétée comme dévalorisante d'un·e client·e ou d'un·e patron·ne:

II [patron] est toujours en train de tout critiquer ce qu'on fait dans le magasin. [...] il est toujours en train de rabaisser ce qu'on fait [...] s'il est énervé, si on croise son chemin, il va se défouler sur nous. Donc il va nous gueuler dessus [...]. Mais bon, je l'ai laissé se calmer, je l'ai laissé gueuler, parce que je sais qu'il est comme ça. Mes collègues, elles m'ont prévenue, je me suis dit je le laisse faire pis après il s'est excusé. (Marianne, apprentie en confiserie)

Dans ce présent exemple, le savoir-faire de métier est développé de manière collective, puisque Marianne en a préalablement discuté avec ses collègues.

La stratégie de passivité devient pour certain·e·s un dernier recours. Plusieurs apprenti·e·s ont en effet d'abord essayé d'agir sur les conditions en entreprise vécues comme difficiles dans le but d'améliorer leur situation ${ }^{4}$. Après de nombreuses tentatives sans véritable succès, la stratégie de passivité peut devenir un moyen pour conserver sa place d'apprentissage. Le contexte de formation de Claudia - apprentie dans un magasin de chaussures - l'illustre bien. Elle a commencé sa formation avec des changements constants de responsables de succursale. Cette situation a miné son encadrement durant son apprentissage. À cela s'ajoute une relation conflictuelle avec sa cheffe. Par la suite, elle a pris des mesures pour changer de lieu de formation en entreprise. Mais sa cheffe, qui voulait la garder, a communiqué à la direction que Claudia ne désirait plus changer de lieu de travail, ce qui a compromis sa démarche. Claudia, dont la relation avec sa cheffe s'est entre-temps améliorée, s'est accommodée de cette situation et essaie d'éviter d'autres conflits, qui rappelle le coping d'évitement, en la côtoyant le moins souvent possible. À cela s'ajoute la pause du matin, pourtant accordée dans le contrat de travail, qui a été supprimée. Ici aussi, même si elle est en désaccord avec cette pratique, Claudia s'est résignée: "Tant pis, on ne fait plus de pause le matin." Elle adopte donc une stratégie de passivité dans le but d'éviter le conflit.

$\overline{4 \quad C f . \text { point } 5.1}$ : Stratégie de résistance. 
Cette stratégie s'explique également par la peur de ne pouvoir terminer sa formation ou de ne trouver un emploi à la fin de l'apprentissage. Cette crainte est apparue de manière évidente en fin de formation pour Karim - apprenti dans une grande enseigne commerciale: "Il y a des fois, j'ai vraiment envie de leur dire "ciao", mais je joue le jeu, tous les matins, je me dis, "mais tu vas bientôt finir, il faut que tu tiennes, sinon tu ne vas jamais arriver où tu veux"." Pourtant plus enclin à adopter la stratégie de résistance, il a également opté pour une stratégie de passivité, car il a conscience qu'un bon certificat de travail est important pour son avenir professionnel. En se souciant de son futur professionnel, il manifeste également la volonté de poursuivre son apprentissage.

Parfois, la stratégie de passivité peut entraîner un isolement des jeunes en formation. Pauline - apprentie en bijouterie - qui s'est beaucoup plainte de sa situation sur le lieu de formation en entreprise s'est progressivement isolée de ses collègues:

Je travaille dans une entreprise où il y a énormément d'hypocrites, on peut faire confiance à personne et ça c'est assez difficile, parce que quand je suis arrivée je faisais confiance à tout le monde et puis, au fur et à mesure, c'est pas tout de suite, on commence à connaître les gens [...] on se rend compte qu'en fait on est tout seul.

Pauline finira par réagir en dernière année dans le but de défendre sa position. Pour elle, le recours à la stratégie de résistance est une conséquence du sentiment de ne plus pouvoir supporter de se taire. Au fil de la formation, elle va acquérir une confiance en elle qui l'incitera à réagir, ce qui à terme lui permettra de s'affirmer.

Mais être toujours diplomate. Pas vexer les gens. Faut aller dans le sens des gens, mais en même temps essayer d'aller dans le nôtre aussi. [...] j'avais de la peine, je disais oui à tout. Mais là maintenant, faut savoir s'affirmer et ne pas faire confiance aux gens. Vraiment pas.

En d'autres termes, une stratégie initiale de passivité peut se transformer en une stratégie de résistance forte à l'égard d'une situation jugée pénible, ce qui va conforter les apprenti·e.s dans leur rapport au métier et au travail.

\subsection{Stratégie d'autonomie: un moyen de renforcer son développement personnel}

La troisième stratégie qui relève également d'un mécanisme d'adaptation tend à une certaine autonomie des apprenti-e.s par la prise de conscience et/ou la valorisation des savoir-faire et savoir-être acquis à travers les tâches effectuées. En réinterprétant les conditions de travail et de formation parfois difficiles comme un défi personnel, les apprenti·e·s, souvent en quête de reconnaissance, vont chercher à s'affirmer. Donner du sens aux différentes tâches qu'elles et ils exécutent dans des conditions de travail données leur permet de mieux vivre leur apprentissage, ce qui contribue à leur développement personnel et professionnel. Les jeunes en formation vont pour ce faire adopter un 
changement comportemental dans le but de mieux maîtriser leur situation d'apprentissage. La stratégie d'autonomie est complexe et peut engendrer chez certain.e-s apprenti.e.s une prise d'initiative et de conscience ou une valorisation de leurs savoir-faire et savoir-être. Nous entendons par autonomie, un processus d'autogestion permettant la régulation de ses propres comportements et expériences impliquant un sentiment de liberté, identité, responsabilité et contrôle (Ryan, 1991).

Pour renforcer leur apprentissage de métier, les apprenti-e.s peuvent s'impliquer personnellement dans leur formation en entreprise. Sandra - apprentie dans un magasin de motos - qui déplore un manque d'encadrement, et désirait apprendre le système de facturation, a fini par demander à sa cheffe de pouvoir l'effectuer par elle-même.

Mais dites-moi vraiment comment vous faites? Et puis, elle m'en a imprimé une, et puis j'ai écrit toute la marche à suivre, tout ce qu'il faut pas oublier [...]. Du coup, ben depuis qu'elle a vu que je savais, c'est toujours moi qui fais. Enfin, avec le temps, ben je fais plein de fiches de mémoire, comme ça au moins, même si tout d'un coup j'ai un gros blanc, ben j'ai tout le temps tout avec moi pour au cas où, pouvoir me dépanner.

Ici, Sandra s'est créée sa propre manière d'apprendre et de travailler par l'élaboration d'aide-mémoire.

Cette prise d'initiative se retrouve chez plusieurs apprenti-e.s qui parfois ont le sentiment d'être insuffisamment encadrés. Cela les encourage à se donner des moyens pour apprendre leur métier et développer leurs compétences. Ce type de stratégie favorise le rapport positif au métier tout en donnant de l'importance aux apprenti-e.s au sein de l'entreprise. Leila - apprentie dans un magasin spécialisé en matériel de construction - l'exprime ainsi:

Je faisais le même travail qu'un chef, en première année, donc du coup c'est vrai que ça m'a fait mûrir super vite. Et puis, les autres, ils sont toujours gérés, ils ont trainé tout le temps, tandis que moi j'ai plus dû me débrouiller toute seule.

Leila valorise ainsi son statut par son investissement personnel au sein de l'entreprise.

Pour pallier les conditions de travail difficiles, telles qu'un encadrement déficient, les apprenti-e.s chercheront aussi, plus particulièrement, à donner du sens à leur formation en s'appuyant sur leurs savoir-faire et savoir-être acquis. Amandine - apprentie dans une grande enseigne alimentaire -, qui regrette l'absence de ses responsables sur le lieu de travail, a complètement intégré cette stratégie d'autonomie dans son mode de fonctionnement. Le manque d'encadrement l'a, d'une certaine manière, encouragée à développer son autonomie: "Moi, à partir de six mois d'apprentissage j'étais autonome. À partir de six mois d'apprentissage, on pouvait me laisser seule dans un rayon, je savais gérer." Amandine a ainsi tiré avantage de sa formation en développant notamment une certaine polyvalence. Elle l'exprime ainsi: 


\begin{abstract}
Donc pendant ces six derniers mois, il me mettait vraiment un peu partout, un jour par-ci, un jour par-là [...] donc je regrette pas d'avoir été bouche-trou, parce que ça fait que je suis polyvalente maintenant. Donc vraiment je sais gérer tout dans le magasin, quand j'ai eu mon nouveau gérant c'est moi qui lui ai fait la visite guidée parce que j'étais celle qui connaissait vraiment le mieux le magasin. Je connaissais tous les rayons, je connais à peu près par cour le magasin, je pourrais fermer les yeux je connais l'emplacement de chaque chose.
\end{abstract}

Cet exemple montre comment la stratégie d'autonomie permet d'acquérir de l'indépendance et une plus grande confiance en soi renforçant le sentiment d'appartenance au métier. Le fait d'assumer de multiples tâches ou de pouvoir prendre des initiatives permet d'être davantage impliqué dans sa formation et indirectement son métier, ce qui peut aussi encourager la reconnaissance des apprenti.e.s au sein de l'entreprise comme membre à part entière. En prenant sa formation en main, Amandine parvient grâce à cette stratégie à atténuer les désagréments d'un contexte de formation insuffisamment encadrant et soutenant.

Même si Leila et Amandine se considèrent comme des cas uniques, la deuxième série des groupes focalisés confirme plus largement cette évolution personnelle au cours de la formation. Lorsqu'on leur a demandé si elles et ils ont beaucoup changé, les apprenti-e.s évoquent principalement des traits de caractère tels que: "maturité", "patience", "maîtrise de soi", "être mûr", "savoir s'habituer à d'autres langages", "s'affirmer", "s'endurcir", "autonome", "très rapide", "se débrouiller tout seul" ou encore "prendre sur soi" qui découlerait également de l'expérience de la stratégie de passivité. Cette prise de conscience des savoir-faire et savoir-être acquis au cours de l'expérience de travail montre également l'évolution du parcours de ces apprenti.e.s durant les trois ans de formation.

\title{
6. Discussion
}

Cette contribution a comme objectif d'étudier la façon dont les jeunes en formation répondent aux exigences et contraintes du métier de gestionnaire de commerce de détail. L'analyse des stratégies de coping comme moyen de faire face aux conditions de travail a permis de relever que les apprenti.e.s ne subissent pas uniquement les conditions difficiles de leur métier. Ces stratégies peuvent ainsi même renforcer leur rapport au métier. En cherchant des arrangements pour améliorer leur situation d'apprentissage, elles et ils vont intégrer aussi bien le savoir-faire théorique et pratique que le savoir-être propre au métier. Le rapport au métier que les apprenti-e.s construisent est ainsi étroitement lié à leur manière de faire face aux difficultés rencontrées durant la formation en entreprise. Elles et ils développent trois stratégies - résistance, passivité et autonomie - qui progressivement leur permettent de faire face aux conditions de travail parfois exigeantes. 
La stratégie de résistance, liée au mécanisme défensif, se traduit par une objection à la sensation de mal-être engendrée par des conditions de formation et de travail pénibles ou des situations ressenties comme injustes. Elle procure aux apprenti.e.s le sentiment d'un pouvoir d'action et d'influence. En adoptant cette stratégie, les apprenti·e.s cherchent à se protéger dans le but de mieux supporter les contraintes de formation en entreprise. Ce type de posture corrobore les études de Moreau $(2006 ; 2009)$ et Lamamra et Duc $(2012 ; 2015)$ qui ont également relevé des réactions de résistance de la part des apprenti-e.s particulièrement lors de difficultés rencontrées sur le lieu de formation en entreprise. Même si la plupart de nos exemples de stratégies sont défensives, nous avons pu relever que cette stratégie peut également permettre d'agir sur le problème dans le but de le résoudre ou tout au moins d'en réduire les tensions internes et externes (Bruchon-Schweitzer 2001; Chabrol, 2005). Ces démarches volontaires et impliquées des apprenti-e.s leur permettent de continuer leur formation et aussi souvent d'être davantage ancré.e-s dans leur métier, car elles et ils développent leur savoir-être professionnel en affrontant les conflits et problèmes.

La stratégie de passivité, qui se rattache au mécanisme d'adaptation, implique une prise de distance par rapport aux personnes ou aux situations conflictuelles dans le but de s'y accommoder. Elle se focalise davantage sur l'émotion, mais ne diminue pas pour autant forcément le pouvoir d'action. Elle permet d'atténuer le poids de situations perçues comme difficiles et inhérentes au métier de gestionnaire de commerce de détail. Cette stratégie a une fonction d'autoprotection notamment face à l'attitude négative des client.e.s. Ainsi, les apprenti.e.s développent un savoir-être professionnel qui pourra être utile lorsqu'elles et ils seront confrontéeess à des situations similaires. Le recours à ce type de stratégie a également comme dessein d'éviter le conflit. Se figer dans une telle stratégie risque cependant d'aboutir à un isolement et, à terme, à une distanciation vis-à-vis de leur métier. Si la situation de détachement s'installe, le danger serait un arrêt d'apprentissage comme le confirment Lamamra et Masdonati (2009) dans leur analyse sur le sujet. Le rapport au métier est dès lors perturbé et le futur professionnel des apprenti·e-s serait mis en péril.

La stratégie d'autonomie par la prise d'initiative et la valorisation des tâches effectuées au sein de l'entreprise encourage les jeunes en formation à développer un sentiment de confiance en soi. Ce mode d'investissement professionnel (Osty, 2003) favorise leur construction aussi bien personnelle que professionnelle. La prise d'initiative, en réponse à un encadrement déficient, peut encourager les apprenti.e.s à créer leur propre manière d'apprendre en vue de parfaire leur savoir-faire pratique. Les apprenti.e.s maîtrisent ainsi une situation problématique tout en consolidant leur place au sein de l'entreprise. Trouver du sens dans les exigences du métier permet de mieux vivre les conditions de travail 
difficiles et d'une certaine manière de maîtriser la formation. En acceptant les conditions de travail comme un défi en vue du développement de leur savoir-faire théorique et pratique, les jeunes en formation y trouvent du sens tel un passage nécessaire pour pouvoir réussir sur le plan professionnel. Ce type de stratégie nécessite la maîtrise d'une situation de formation perçue comme défavorable. À travers ce type de stratégie, les apprenti-e.s cherchent aussi de la reconnaissance leur permettant de mieux se positionner et de s'affirmer au sein de l'entreprise.

Alors même qu'aucune stratégie ne peut être définie a priori comme étant efficace pour gérer l'ensemble des circonstances et situations difficiles, ce sera notamment leur combinaison, particulièrement en fin de formation avec le cumul d'expériences, qui permettra une meilleure gestion des situations difficiles favorisant ainsi un rapport positif au métier. Nous rejoignons ici Lazarus et Folkman $(1984 ; 1988)$ qui abordent la question du coping comme un processus s'inscrivant dans une perspective dynamique. Notre analyse va même plus loin puisqu'elle montre qu'une sorte de jeu entre les stratégies est opéré par les apprenti.e.s selon la situation.

La mise en place de ces différentes stratégies ne se fait en revanche pas hors contexte, mais bien dans le cadre de l'entreprise lors de difficultés rencontrées. La stratégie de résistance adoptée par les apprenti-e·s a une chance d'être appliquée, si elle est prise en compte par les collègues et l'employeur, pour autant qu'elle puisse s'élaborer et créer ainsi un effet positif sur leur rapport au métier. Se distancer d'une situation difficile par une stratégie de passivité apparaît souvent comme une stratégie répondant à un manque d'écoute des besoins des apprenti.e.s avec le risque d'un désinvestissement de leur part. Enfin, la stratégie d'autonomie est facilitée par la reconnaissance et la valorisation des savoir-faire et savoir-être développés par les apprenti-e.s. Si les jeunes en formation ne sont pas reconnus, il y a un risque qu'elles ou ils se sentent exploitée.es, ce qui remet en question le rapport positif qui a pu être développé à l'égard de l'entreprise et/ou du métier (Caprani, Duemmler et Felder, 2019).

En conclusion, l'intérêt pour la pratique de définir ces stratégies est de permettre aux personnes formatrices en entreprise et aux enseignant.e.s d'être plus attentifs aux problèmes et stratégies des apprenti-e.s et si nécessaire de réajuster leur propre encadrement et enseignement. L'élaboration d'outils pédagogiques qui s'appuieraient sur ces stratégies pourrait travailler sur certains points problématiques tels que la gestion de client·e-s difficiles, des conflits avec un·e supérieur·e ou le développement de l'autonomie. La discussion de ces stratégies avec des apprenti-e.s serait également un moyen de prise de conscience et ainsi d'appréhender autrement les difficultés sur le lieu de travail. 


\section{Références}

Angeloff, T. (2000). Le temps partiel, un marché de dupes? Paris: Syrios.

Askenazy, P., Behaghel, L., Laouenan, M. et Meurs, D. (2019). Quarante ans d'analyse du travail et de l'emploi: points de vue de quatre économistes, Travail et Emploi, 158. Repéré à https:// www.cairn.info/revue-travail-et-emploi-2019-2-page-69.htm

Barbier, P. (2012a). Travailler à contretemps. Vendre le soir, le dimanche et les jours fériés dans les grands magasins. Temporalités, 16.

Barbier, P. (2012b). Contrainte relationnelle et résistance au travail. Les vendeurs des grands magasins. Sociétés contemporaines, 86(2), 31-57.

Bauman, Z. (1998). Work, Consumerism and the New Poor. Buckingham: Open University Press.

Benoun, M. (2015). Le commerce de détail en Suisse. Lausanne: Presses polytechniques et universitaires romandes.

Billett, S. (2006). Work, Change and Workers. Dordrecht: Springer.

Billings, A. G. et Moos, R. H. (1981). The role of coping responses and social resources in attenuating the impact of stressful live events. Journal of Behavioural Medicine, 4, 139-157.

Bourdieu, P. (1980). Le sens pratique. Paris: Minuit.

Bozkurt, Ö. et Grugulis, I. (2011). Why retail work demands a closer look? Dans I. Grugulis, C. Lloyd, C. Smith et C. Warhurst (dir.), Retail Work (p. 1-24). Londres: Palgrave Macmillan.

Bruchon-Schweitzer, M. (2001). Le coping et les stratégies d'ajustement face au stress. Recherche en soins infirmiers, 67(12), 68-83.

Caprani, I., Duemmler, K. et Felder, A. (2019). Construire une identité professionnelle malgré des conditions de travail difficiles: les apprenti·e·s en commerce de détail en suisse. Éducation et socialisation, 53. https://doi.org/10.4000/edso.7144

Chabrol, H. (2005). Les mécanismes de défense. Recherche en soins infirmiers, 82(3), 31-42.

Charmaz, K. (2001). Qualitative interviewing and grounded theory analysis. Dans J. F. Gubrium et J. A. Holstein (dir.), Handbook of Interview Research. Context and Methods (p. 675-694). Thousand Oaks, CA: Sage Publications.

Cianferoni, N. (2019). Travailler dans la grande distribution. La journée de travail va-t-elle redevenir une question sociale? Zurich/Genève: Seismo.

Colley, H., James, D., Diment, K. et Tedder, M. (2003). Learning as becoming in vocational education and training: class, gender and the role of vocational habitus. Journal of Vocational Education Training, 55(4), 471-498.

Coutrot, T. et Mermilliod, C. (2010). Les risques psychosociaux au travail: les indicateurs disponibles. Dares Analyses, 81.

Coyne, J. C., Aldwin, C. et Lazarus, R. S. (1981). Depression and coping in stressful episodes. Journal of Abnormal Psychology, 90, 439-447.

Dantchev, N. (1989). Stratégies de coping et pattern A coronarogène. Revue de médecine psychosomatique, 17-18, 21-30.

Dantzer, R. (1989). L'illusion psychosomatique. Paris: Odile Jacob.

Duemmler, K., Felder, A. et Caprani, I. (2016). Flexibility and autonomy demands: Retail apprentices' sens-making and professional identities. Communication présentée à la European Conference on Education Research (ECER). Conference Proceeding, Dublin, Irlande.

Endler, N. S. (1988). Hassles, health and hapiness. Dans M. P. Janisse (dir.), Individual Differences, Stress and Health Psychology (p. 24-56). New York, NY: Springer. 
Endler, N. S. et Parker, J. A. (1990). Multidimensional assessment of coping : A critical evaluation. Journal of personality and social psychology, 58, 844-854.

Folkman, S. et Lazarus, R. S. (1980). An analysis of coping in a middle-aged community sample. Journal of Health and Social Behavior, 21, 219-239.

Folkman, S. et Moskowitz, J. T. (2004). Coping: Pitfalls and promise. Annual Review of Psychology, 55(1), 745-774.

Gadrey, N., Jany-Catrice, F. et Pernod-Lemattre, M. (2005). Les conditions de travail des employés non qualifiés. Dans D. Méda et F. Vennat (dir.), Le travail non qualifié. Permances et paradoxes (p. 182-196). Paris: La Découverte.

Gatta, M. (2011). In the "blink" of an eye - American high-end small retail businesses and the public workforce system. Dans I. Grugulis, C. Lloyd, C. Smith et C. Warhurst (dir.), Retail Work (p. 49-67). Londres: Palgrave Macmillan.

Glaser, B. et Strauss, A. L. (1967). The Discovery of Grounded Theory. Chicago, IL: Aldine.

Lamamra, N. et Duc, B. (2012). La transition école-travail comme moment de socialisation professionnelle? Le rapport au travail des apprenti.e.s suisses ayant connu un arrêt prématuré de leur formation. Chroniques du travail, 2, 28-47.

Lamamra, N. et Duc, B. (2015). Die duale Berufsausbildung. Von einem sanften Übergang zur Arbeitswelt zu einer Anpassung an ihre neue Mechanismen. Dans K. Häfeli, P. Markus, P. Neuenschwander et S. Schumann (dir.), Berufliche Passagen im Lebenslauf (p. 101-126). Cham: Springer.

Lamamra, N. et Madonati, J. (2009). Arrêter uneformation professionnelle. Mots et maux d'apprenti.e.s. Lausanne: Antipodes.

Lazarus, R. et Folkman, S. (1984). Stress, Appraisal and Coping. New York, NY: Springer.

Lazarus, R. et Folkman, S. (1988). The relationship between coping and emotion. Social and Scientific Medicine, 26, 309-317.

Lazarus, R. et Launier, R. (1978). Stress-related transactions between person and environment. Dans L. A. Pervin et M. Lewis (dir.), Perspectives in Interactional Psychology (p. 287-327). New York, NY: Plenum.

Mariage, A. (2001). Stratégies de coping et dimensions de la personnalité: étude dans un atelier de couture. Le travail humain, 64(1), 45-59.

Mercure, D. et Vultur, M. (2010). La signification du travail. Nouveau modèle productif et ethos du travail au Québec. Québec, QC: Presses de l'Université Laval.

Meyer, T. et Bertschy, K. (2011). The long and winding road from education to labour market: The TREE cohort six years after leaving compulsory school. Dans M. M. Bergman, S. HupkaBrunner, A. Keller, T. Meyer et B. E. Stalder (dir.), Transitions juvéniles en Suisse. Résultats de l'étude longitudinale TREE. Youth transitions in Switzerland (p. 92-119). Zurich: Seismo.

Moreau, G. (2006). Le monde des apprentis. Les dossiers insertion, éducation et société, 178.

Moreau, G. (2009). Affiliations et désaffiliations aux métiers de la mécanique automobile: le cas des apprentis. CPC documents, 1.

Nicchi, S. et Le Scanff, C. (2005). Les stratégies de faire face. Bulletin de psychologie, 58(1), 97-100.

Osty, F. (2003). Le désir de métier. Engagement, identité et reconnaissance au travail. Rennes: Presses Universitaires de Rennes.

Park, C. et Folkman, S. (1997). Meaning in the contexte of stress and coping. Review of General Psychology, 1, 115-144.

Paulhan, I. (1992). Le concept de coping. L'année psychologique, 92(4), 545-557. 
Pearlin, L. I. et Schooler, C. (1978). The structure of coping. Journal of Health and Social Behavior, 19, 2-21.

Price, R. (2011). Technological change, work re-organisation and retail workers' skills in production-oriented supermarket. Dans I. Grugulis, C. Lloyd, C. Smith et C. Warhurst (dir.), Retail Work (p. 88-105). Londres: Palgrave Macmillan.

Quiénnec, Y., Gadbois, C. et Preteur, V. (1995). Souffrir de ses horaires de travail: poids de l'âge et histoire de vie. Dans J.-C. Marquié, D. Paumès et S. Volkoff (dir.), Le travail au fil de l'âge (p. 277-304). Toulouse: Octarès.

Ray, C., Lindop, J. et Gibson, S. (1982). The concept of coping. Psychological Medicine, 12, 385-395.

Roberts, S. (2012). Gaining skills or just paying the bills? Workplace learning in low-level retail employment. Journal of Education and Work, 26(3), 267-290.

Ryan, R. M. (1991). The nature of the self in autonomy and relatedness. Dans J. Strauss et G. R. Goethals (dir.), The Self: Interdisciplinary Approaches. New York, NY: Springer.

Salvisberg, A. (2012). Mechanismen des Arbeitsmarktgeschehens aus soziologischer Perspektive: theoretische Überlegungen und empirische Beispiele aus der Berufseinstiegsphase. Dans M. Bergmann, S. Hupka-Brunner, T. Meyer et S. Robin (dir.), Bildung - Arbeit - Erwachsenwerden. Ein interdisziplinärer Blick auf die Transitionen im Erwachsenenalter (p. 133-157). Wiesbaden: Springer VS.

SEFRI (2020). La formation professionnelle en Suisse. Faits et données chiffrées 2020. Berne: Secrétariat d'État à la formation, la recherche et l'innovation.

Suls, J. et Fletcher, B. (1985). The relative efficacy of avoidant and non-avoidant coping strategies: A meta analysis. Health Psychology, 4, 249-288.

Tomlinson, M. (2013). Education, Work and Identity. Themes and Perspectives. Londres: Bloomsbury.

UNIA (2012). Die Branche Detailhandel. Analyse, Ziele und Forderungen der Unia. Berne: Gewerkschaft Unia.

Voirol-Rubido, I. et Hanhart S. (2015). Face aux mutations des marchés de l'emploi, quelles politiques de formation? Revue française de pédagogie, 192(3), 5-10.

Voss, G. G. (2002). Auf dem Weg zum Individualberuf? Zur Beruflichkeit des Arbeitskraftunternehmers. Dans T. Kurz (dir.), Der Beruf in der Moderne (p. 287-314). Opladen: Leske und Budrich.

Williams, C. et Connell, C. (2010). Looking good and sounding right: Aesthetic labor and social inequality in the retail industry. Work and Occupations, 37(3), 349-377. 